\begin{tabular}{|c|l|}
\hline Title & Blooming theory of tristearin \\
\hline Author(s) & Mayama, Hiroyuki \\
\hline Citation & $\begin{array}{l}\text { Soft Matter, 5(4), 856-859 } \\
\text { https:/doi.org/10.1039/6815005b }\end{array}$ \\
\hline Issue Date & 2009-02-21 \\
\hline Doc URL & http://hdl.handle.net/2115/42651 \\
\hline Rights & Soft Matter, 2009, 5, 856-859- Reproduced by permission of The Royal Society of Chemistry (RSC) \\
\hline Type & article (author version) \\
\hline File Information & Mayama_SoftMatter.pdf \\
\hline
\end{tabular}

Instructions for use 


\title{
Blooming theory of tristearin
}

\section{Hiroyuki Mayama}

\author{
Received (in $X X X, X X X) X$ th $X X X X X X X X X 200 X$, Accepted $X$ th $X X X X X X X X X 200 X$ \\ First published on the web $X$ th $X X X X X X X X X 200 X$ \\ ${ }_{5}$ DOI: 10.1039/b000000x
}

\begin{abstract}
"Blooming" of tristearin (SSS), a spontaneous formation of flake-like surface structure on a triacylglycerols (TAG), was discussed. It has been well known that blooming of TAGs was closely related to the polymorphism, however, a foundamental question on how the blooming on micrometer scale was connected with the polymorphism on molecular scale is still unclear. We 10 theoretically found that the flakes were formed to release deformation energy of surface induced by phase transformation from the $\alpha$-phase to the $\beta^{\prime}$-phase in the polymorphism of SSS. It was also able to explain the flake size observed in experiments $(\sim 3 \mu \mathrm{m} \times 3 \mu \mathrm{m} \times 0.1 \mu \mathrm{m})$. This theory would provide a better understanding of the spontaneous formation of surface structures in chocolate, fats, fatty acids, waxes, and water-repellent leaves of grasses.
\end{abstract}

\section{${ }_{15}$ 1. Introduction}

"Blooming" or "Fat Blooming" is a famous phenomenon in chocolate. ${ }^{1}$ White spots are sometimes seen on chocolate which has been stored at high temperature. By scanningelectron-microscope (SEM) observation, many fine flakes, 20 like petals of flowers, can be seen in the white spots, ${ }^{2}$ which is why it is called "blooming". It is well known that the blooming of chocolate is related to the polymorphism (spontaneous phase transformations) of triacylglycerols (TAGs) in cocoa butter (CB), ${ }^{2-7}$ however, a fundamental ${ }_{25}$ question how the blooming is related to the polymorphism is still unclear in spite of intensive studies on the polymorphism and crystal structures of $\mathrm{CB}^{3-7}$ There are some reasons. One is a complexity of CB because it is a mixture of SSS, tripalmitin (PPP), 1,3-distearoyl-2-oleoylglycerol (SOS), and so on. ${ }^{1}$ 30 Therefore, determinations of crystalline phases and crystal structures are very difficult. Other may be a big scale gap between blooming and polymorphism. Micrometer-scale flakes are formed in blooming, whereas phase transformations occur in molecular scale.

${ }_{35}$ Relation to this, we have obtained clear experimental results that the blooming of TAGs and a wax with polymorphism was essential for the spontaneous formation of fractal super waterrepellent surfaces ${ }^{8-11}$ and the preparation of a fractal body (fractal porous silica). ${ }^{12,13}$ For example, fine flakes which 40 increase the surface area play a significant role in the fractal super water-repellent surfaces of hydrophobic materials, where the thickness of the flakes determines the lower cutoff of the scale range in which the surface fractal dimension $D_{\mathrm{s}} \sim$ 2.3 appeared. ${ }^{8,9}$ Thus, blooming is important not only in ${ }_{45}$ chocolate, but also in related phenomena.

The purpose of this article is to unveil the blooming mechanism of SSS. SSS was selected as a model TAG to investigate the correlation between blooming and polymorphism because it is one of the most intensively ${ }_{50}$ studied TAGs with regard to both its phase behaviour and the crystal structures in polymorphism. ${ }^{14-19}$ We will present the blooming mechanism of SSS based on the phase transformations.

\section{Experiments}

${ }_{55}$ SSS (m.p. $=73.5^{\circ} \mathrm{C}$ ) was purchased from Tokyo Chemical Industries Co., Ltd., and used without purification. Sodium dodecyl sulfate (SDS) was obtained from Wako Pure Chemical Industries, Ltd. Salad oil was used as rapeseed oil. SSS samples were melted on cover glasses (Matsunami Glass ${ }_{60}$ Co., Ltd.) and quenched for solidification in air. To compare the surface structures before and after blooming, two kinds of SSS samples were prepared by temperature treatment: one stood at $65{ }^{\circ} \mathrm{C}$ for 4 days to induce the blooming completely, ${ }^{11}$ and the other was solidified before scanning-electron65 microscopic (SEM) observations. Addition to them, other solidified samples were immersed into a $1 \mathrm{~mol} / \mathrm{l}$ SDS solution and rapeseed oil, and stood at $40{ }^{\circ} \mathrm{C}$ for 2 days to check the effect of liquid-solid interfacial energy. The surfaces of the SSS samples were observed using a JEOL JSM-7400F 70 scanning electron microscope (SEM), at the open facility of Creative Initiative "Sosei" (CRIS), Hokkaido University.

\section{Results and discussion}

Figure 1 shows typical SEM images of the surface of SSS before and after blooming. The surface was smooth before the 75 blooming as shown in Figs. 1 (a) - (c), but was covered by fine flakes after the blooming as shown in Figs. 1 (d) - (f).
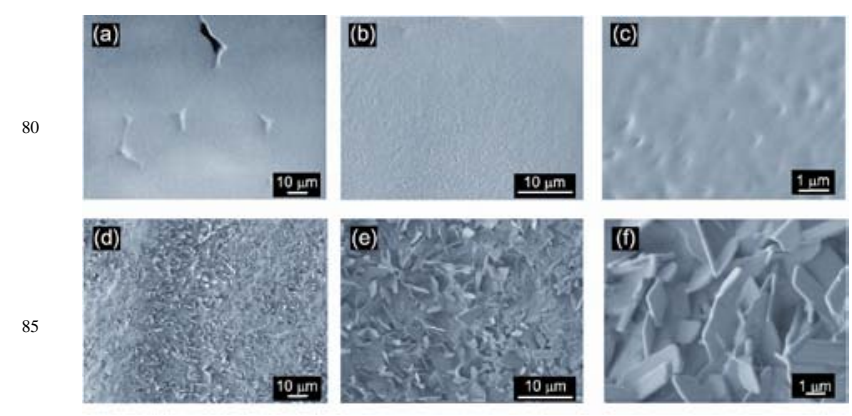

Fig. 1 SEM images at different magnifications of the surfaces of tristearin (SSS) before (a)-(c) and after (d)-(f) blooming in air. 

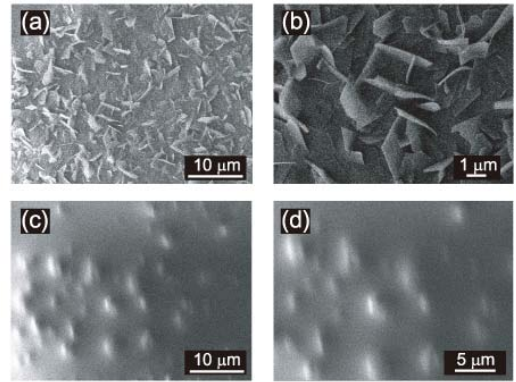

Fig. 2 SEM images at different magnifications of the surface of the SSS samples immersed in a $1 \mathrm{~mol} / \mathrm{l}$ SDS solution (a) - (b) and in a rapeseed oil (c) - (d) stood at $40{ }^{\circ} \mathrm{C}$ for 2 days.

(a)

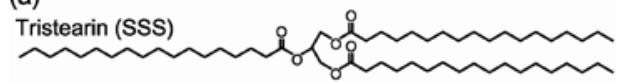

(b)

Fig. 3 (a) Molecular structure of SSS. (b) Rough scheme of phase transformations in the polymorphism of SSS in terms of the crystal structures of bilayers14-18. There are two paths to reach the $\beta$-phase, the stable state. The first is from the $\alpha$-phase (meta-stable) formed by 40 quenching through the $\beta$ '-phase (meta-stable) to the $\beta$-phase (stable state). The other path is "tempering", or the direct path from the liquid state to the $\beta$-phase which is achieved by careful treatment.

From Fig. 1 (f), the typical size of the flake is roughly $\sim 3$ $\mu \mathrm{m} \times 3 \mu \mathrm{m} \times 0.1 \mu \mathrm{m}$. Similar surface structures have been ${ }_{45}$ observed in chocolate, ${ }^{2}$ other TAGs $^{8}$ and wax. ${ }^{9}$

Figure 2 illustrates typical SEM images of the SSS samples immersed in the SDS solution and the rapeseed oil to check the effect of liquid-solid interfacial energy on the blooming. As shown in Figs. 2 (a) and (b), fine flakes were formed in the ${ }_{50}$ SDS solution and then the flake size was almost same. On the other hand, many humps were observed on the surface of the SSS immersed in rapeseed oil as shown in Figs. 2 (c) and (d). The typical size was $3 \mu \mathrm{m} \times \leq 1 \mu \mathrm{m}$, which is good agreement with the size of the cross section of the flakes. Since the 55 rapeseed oil includes various TAGs, it is expected that the humps are imprints of the flakes dissolved into the rapeseed oil stored at $40{ }^{\circ} \mathrm{C}$. These demonstrate that the blooming can not be affected by the liquid-solid interfacial energy.
Therefore, the origin of the blooming should be found into the 60 polymorphism of SSS.

Figure 3 summarizes the polymorphism of SSS. Since SSS has three long alkyl chains as shown in Fig. 3 (a), it shows a tuning-fork conformation in the liquid and solid states. ${ }^{20,21}$ Figure 3 (b) illustrates phase transformations in the ${ }_{65}$ polymorphism of SSS in terms of the bilayer. ${ }^{14-19}$ There are two paths to reach the $\beta$-phase from the liquid state. One is through the $\alpha$-phase and $\beta$ '-phase to the $\beta$-phase. Upon quenching, individual SSS bilayers are immediately formed as the $\alpha$-phase, which changes to the $\beta$ '-phase, and finally to the

$70 \beta$-phase. Roughly, the phase transformations of SSS are accompanied by the tilting of SSS molecules in the bilayer. Thus, the bilayers are deformed in the phase transformations as if they are pressed. The other path is the direct path from the liquid state to the $\beta$-phase: "tempering"., ${ }^{1,8}$ We can discuss 75 the blooming based on the deformation energies of the bilayers. Then the surface energy of the flake must also be considered. Figure 3 shows a rough schematic representation of the blooming. We consider an imaginary flake with width $l$, depth $d(=l)$ and height $h$ on the surface before the blooming. ${ }_{80}$ We assume that the SSS molecules in the vicinity of the surface at solidification would be stacked perpendicular to the surface, as shown in Fig. 4 (a), to decrease the surface energy by the $\mathrm{CH}_{3}$ groups at the end of the alkyl chains. We also assumed that the spontaneous formation of a flake could be 85 divided into two processes because the boundaries between individual SSS bilayers could act as planar defects. Therefore, a crack would first be propagated along the boundary as shown in Fig. 4 (b), and peeling of a flake, including several SSS bilayers, would then occur as shown in Fig. 4 (c). In go other words, the blooming is achieved to release the deformation energy.

Let us consider the blooming in this scenario. In the first process, from Fig. 4 (a) to Fig. 4 (b), the deformation energy of the flake $E_{\mathrm{d}}$ would be

95

$$
E_{d}=\frac{1}{2} E \varepsilon^{2} l^{2} h
$$

where $E$ is Young's modulus and $\varepsilon=\Delta h / h, \Delta h$ is the change in thickness. On the other hand, the surface energy of the two surfaces generated by the crack $E_{\mathrm{s}}$ is

$$
E_{s}=2 \gamma^{2},
$$

100 where $\gamma$ is the surface tension. $E_{\mathrm{d}}$ would be roughly equal to $E_{\mathrm{s}}$ for the crack formation. From $E_{\mathrm{d}} \approx E_{\mathrm{s}}, h$ can be obtained as

$$
h \approx \frac{4 \gamma}{E \varepsilon^{2}} .
$$

In the second transformation, from Fig. 4 (b) to Fig. 4 (c), the flake is peeled from the surface together with the generation 105 of new cross-sections. The deformation energy of the flake arising from the increase in width $E_{\mathrm{d}}{ }^{\prime}$ is 


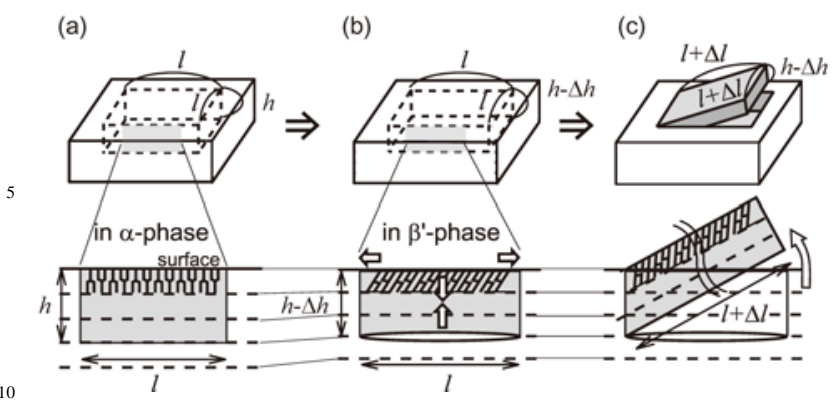

Fig. 4 Rough scheme of the spontaneous formation of a flake with phase transformation in terms of the tilting of SSS molecules in individual bilayers. The phase transformation from the $\alpha$-phase to the $\beta$ '-phase is shown as an example. The initial phase is the $\alpha$-phase in the first phase

15 transformation and the $\beta$ '-phase in the second phase transformation. (a) The height and width of an imaginary flake on the surface are $h$ and $l$, respectively. (b) With the tilting of SSS molecules, the height is reduced and a crack develops along the boundary and width is extended. (c) The flake peels from the surface.

$$
E_{d}^{\prime}=\frac{1}{2} E\left(\varepsilon^{\prime}\right)^{2} l^{2}(h-\Delta h),
$$

20 where $\varepsilon^{\prime}=\Delta l / l=-\sigma(\Delta h / h)=-\sigma \varepsilon, \sigma$ is Poisson's ratio, and $\Delta l$ is the change in the width of the flake due to the phase transformation. On the other hand, the surface energy is due to the new cross-sections. To distinguish it from $E_{\mathrm{s}}$ in the first transformation, the surface energy is denoted as $E_{\mathrm{S}}{ }^{\prime}$ :

25

$$
E_{S}^{\prime}=6 \gamma^{\prime} l(h-\Delta h),
$$

where the factor 6 is the number of new surfaces, as shown in Fig. 4 (c), and $\gamma$ is due to the new surfaces. To peel the flake from the surface, $E_{\mathrm{d}}{ }^{\prime} \approx E_{\mathrm{s}}{ }^{\prime}$. As a result, $l$ can be obtained as

$$
l \approx \frac{12 \gamma^{\prime}}{E\left(\varepsilon^{\prime}\right)^{2}}
$$

30 Thus, the flake size formed after peeling is roughly $\sim \times l \times h$. Let us evaluate the theoretical flake size of SSS based on the phase transformations. To obtain theoretical values of $t$ and $l$, the values of $\gamma, \gamma, E, \varepsilon, \sigma$ and $\varepsilon^{\prime}$ were obtained as follows. $\gamma$ was obtained from the surface tension of tristearin $(\sim 30 \mathrm{mN}$ $35 \mathrm{~m}^{-1}$ in liquid at $\left.60{ }^{\circ} \mathrm{C}\right)^{22}$ and it was assumed that $\gamma=\gamma \cdot \varepsilon$ was estimated to be $\sim-0.07$ from the change in the thickness. $E$ was obtained from the relation $G=E / 2(1+\sigma)$, where $G$ is the shear modulus and $\sigma$ is Poisson's ratio. The value of $G$ was obtained from that of paraffin $\left(G=10^{8} \mathrm{~Pa}\right.$ in solid, m.p. $=$ $4057.5^{\circ} \mathrm{C}$ ), ${ }^{23}$ while $\sigma$ was estimated to be $\sim 0.3 .{ }^{24}$ From $\varepsilon$ and $\sigma$, $\varepsilon^{\prime} \sim 0.02$. With $G$ and $\sigma, E$ was estimated to be $2.6 \times 10^{8} \mathrm{~Pa}$. Based on the values of $\gamma \sim \gamma \sim 30 \mathrm{mN} \mathrm{m}^{-1}, E \sim 2.6 \times 10^{8} \mathrm{~Pa}, \varepsilon$ $\sim 0.07$ and $\varepsilon^{\prime} \sim 0.02$, the theoretical flake size in the first phase transformation was $h \sim 0.1 \mu \mathrm{m}$ and $l \sim 3 \mu \mathrm{m}$.

${ }_{45} \mathrm{Next}$, we would like to calculate the flake size in the second transformation. It was assumed that the values of $\gamma, \gamma, E$ and $\sigma$ were the same. From the change in thickness, $\varepsilon \sim-0.04$. As a result, $h \sim 0.27 \mu \mathrm{m}$ and $l \sim 10 \mu \mathrm{m}$ were obtained. Since the flake size in the first transformation is smaller than this flake 50 size, peeling of a flake from the flake formed in the first transformation is impossible. Thus, it is found that the blooming of SSS is induced in the phase transformation from the $\alpha$-phase to the $\beta$ '-phase. Actually, it has found that the bloomed surface was not obtained by the tempering in 55 alkylketene dimer (AKD), a blooming material. ${ }^{8}$

Very surprisingly, the theoretical flake size in the first transformation is very close to the actual size $(\sim 3 \mu \mathrm{m} \times 3$ $\mu \mathrm{m} \times 0.1 \mu \mathrm{m})$. Even if precise values of $\gamma, \gamma, E$ and $\sigma$ are used, the theoretical flake size would be almost the same. 60 Addition to this, we considered isotropic deformation in terms of $\sigma$ to simplify the theoretical treatment. Since the actual deformation of the crystalline structure from the $\alpha$-phase to the $\beta$ '-phase is somewhat complex, ${ }^{14} \sigma$ is anisotropic, which explains why long flakes are formed. This scenario also 65 explains that why the surface of SSS was completely covered by fine flakes after the blooming as shown in Figs. 1 (d)-(f) was due to the release of the deformation energy.

\section{Conclusion}

The relationship between the blooming and the polymorphism 70 of SSS was first explained in terms of the deformation energy of the flake and the surface energy. This blooming theory also explains not only the blooming of SSS, but also the blooming in other TAGs and waxes such as trimyristin (MMM), tripalmitin (PPP), distearin, 1,3-distearoyl-2-oleoylglycerol 75 (SOS) and AKD because they also show blooming with polymorphism. ${ }^{7,8,10,11}$ This will be clarified in future studies. Here, it should be noted that the blooming theory is also helpful in experiment. For example, one would be able to controle the flake size through the surface energy and the 80 phase transformations. Not only the flake size, the kinetics of blooming is also essential in the experimental designs. ${ }^{10,11}$ Very recently, we have found melting point lowering with the blooming by the mixing of stearin into SSS though the detail is skipped, where the kinetics became faster with descending 85 melting point. Thus, it is possible to change the kinetics by the incorporation of impurities. These experimental strategies would be developed for future useful applications; e.g., super liquid-repellent materials, printing blocks for micro-printing processes, low ahdesion surfaces, etc. Moreover, this 90 blooming theory may explain how super water-repellent leaves of grasses are spontaneously formed. ${ }^{25-27}$

\section{Acknowledgement}

The author thanks Prof. Kaoru Tsujii and Mr. Takayuki Minami, Nanotechnology Research Center, Research Institute 95 for Electronic Science, Hokkaido University for fruitful discussions and comments. This work was supported by a Grant-in-Aid for Young Scientists (B) (Grant No. 18710089) from the Ministry of Education, Culture, Sports, Science and Technology, Japan (MEXT).

\section{${ }_{100}$ Notes and references}

1 S. T. Beckett, The Science of Chocolate, Royal Society of Chemistry, London, 2008.

2 D. Rousseau and P. Smith, Soft Matter, 1998, 4, 1706-1712. 
3 K. Sato and T. Koyano, Chapter 12, in Crystallization Process in Fats and Lipid Systems, eds. N. Garti and K. Sato, Marcel Dekker, New York, 2001.

4 A. G. Marangoni and S. E. McGauley, Cryst. Growth Design, 2003, 3, 95-108.

5 R. Peschar, M. Pop, D. J. A De Ridder, J. B. van Mechelen, R. A. J. Driessen, and H. Schenk, J. Phys. Chem. B, 2004, 108, 1545015453.

6 S. D. MacMillan and K. J. Roberts, Cryst. Growth Design, 2002, 2, 221-226.

7 J. B. van Mechelen, R. Peschar and H. Schenk, Acta Cryst. B, 2006, 62, 1121-1130.

8 T. Minami, H. Mayama, S. Nakamura, S. Yokojima, J. -W. Shen, and K. Tsujii, Soft Matter, 2008, 4, 140-144.

159 T. Onda, S. Shibuichi, N. Satoh, and K. Tsujii, Langmuir, 1996, 12, 2125-2127.

10 W. Fang, H. Mayama and K. Tsujii, Colloids Surf. A, 2008, 316, 258265.

11 W. Fang, H. Mayama and K. Tsujii, J. Phys. Chem. B, 2007, 111, 564-571.

12 H. Mayama and K. Tsujii, J. Chem. Phys., 2006, 125, 124706.

13 D. Yamaguchi, H. Mayama, S. Koizumi, K. Tsujii, and T. Hashimoto, Eur. Phys. J. B, 2008, 63, 153-163.

14 L. Hernqvist, Chapter 3, in Crystallization and Crystallization of Fats 25 and Fatty Acids, eds. N. Garti and K. Sato, Marcel Dekker, New York, 1988.

15 D. R. Kodali, D. Atkinson, T. G. Redgrave, and D. M. Small, J. Lipid Res., 1987, 28, 403-413.

16 J. W. Hagemann and W. H. Tallent, J. Am. Oil Chem. Soc., 1972, 49, 118-123.

17 R. E. Timms, Prog. Lipid Res., 1984, 23, 1-38.

18 E. S. Lutton and A. J. Fehl, Lipids, 1969, 5, 90-99.

19 A. van Langevelde, R. Peschar, and H. Schenk, Acta Cryst. B, 2001, 57, 372-377.

3520 P. T. Callaghan, Chem. Phys. Lipids, 1977, 19, 56-73.

21 P. T. Callaghan and K. W. Jolley, Chem. Phys. Lipids, 1972, 23, 133142.

22 J. J. Jasper, J. Phys. Chem. Ref. Data, 1972, 1, 841-1009.

23 Nihon Kagakukai, Kagakubinran Kiso-hen. pp. II-31, Maruzen,

$40 \quad$ Tokyo, 1993 (in Japanese).

24 The $\sigma$ of SSS was estimated as follows: extensible materials such as gold, silver and rubber show $\sigma=0.37 \sim 0.49$, while metals such as iron and copper show $\sigma \sim 0.3$, polyethylene shows $\sigma \sim 0.34$ and glass shows $\sigma \sim 0.2$. From these values, we expected that $\sigma$ for SSS was between those for polyethylene and glass, but would be closer to that fo polyethylene than that for glass because SSS is somewhat elastic. Thus, we assumed $\sigma \sim 0.3$ for SSS. In addition, since $l \propto \gamma^{\prime} / G\left(\sigma^{2}+\sigma^{3}\right) \approx \gamma^{\prime} / G \sigma^{2}$ for $\sigma^{3}<<1$, it is obvious that the order of $l$ does not change so much around $\sigma \sim 0.3$.

5025 W. Barthlot and C. Neinhuis, Planta, 1997, 202, 1-8.

26 A. A. Millar, S. Clemene, S. Zachgo, E. H. Giblin, and D. C. Taylor, Plant Cell, 1999, 11, 825-838.

27 D. Post-Beittenmiller, Ann. Rev. Plant Physiol Plant Mol. Biol., 1996, 47, 405-430.

55

Nanotechnology Research Centre, ResearchInstitute for Electronic Science, Hokkaido University, Sapporo 001-0021, Japan. Fax: +81-11706-9346; Tel:+ 81-11-706-9346; E-mail:mayama@es.hokudai.ac.jp 